\title{
Hyperspectral Imaging Based on Compressive Sensing: Determining Cancer Margins in Human Pancreatic Tissue ex Vivo, a Pilot Study
}

\author{
Joseph Peller ${ }^{1}$, Cobey L. McGinnis' ${ }^{1}$, Kyle J. Thompson ${ }^{2}$, Imran Siddiqui' ${ }^{2}$, John Martinie ${ }^{2}$, \\ David A. Iannitti ${ }^{2}$, Susan R. Trammell ${ }^{1}$
}

${ }^{1}$ Department of Physics and Optical Science, University of North Carolina at Charlotte, Charlotte, USA

${ }^{2}$ Carolinas Medical Center, Charlotte, USA

Email: srtramme@uncc.edu

How to cite this paper: Peller, J., McGinnis, C.L., Thompson, K.J., Siddiqui, I., Martinie, J., Iannitti, D.A. and Trammell, S.R. (2021) Hyperspectral Imaging Based on Compressive Sensing: Determining Cancer Margins in Human Pancreatic Tissue ex Vivo, a Pilot Study. Open Journal of Medical Imaging, 11, 115-131.

https://doi.org/10.4236/ojmi.2021.114011

Received: October 7, 2021

Accepted: December 4, 2021

Published: December 7, 2021

Copyright $\odot 2021$ by author(s) and Scientific Research Publishing Inc. This work is licensed under the Creative Commons Attribution International License (CC BY 4.0).

http://creativecommons.org/licenses/by/4.0/

\begin{abstract}
Cancer is the second-leading cause of death in the United State and surgery remains the primary treatment for most solid mass tumors. However, accurately identifying tumor margins in real-time remains a challenge. In this study, the design and testing of hyperspectral imaging (HSI) system based on a single-pixel camera engine is discussed. The primary advantage of a single pixel architecture over traditional scanning HSI techniques is its high sensitivity and potential to function at low light levels. The objective for the imaging system described here is to detect changes in the reflectance spectra of tissue and to use these differences to delineate tumor margins. This paper presents the results of a 19-patient pilot study that assesses the ability of the HSI system to use reflectance imaging to delineate adenocarcinoma tumor margins in human pancreatic tissue imaged ex vivo. Pancreatic tissue excised during pancreatectomy was imaged immediately after being sent to the pathology lab. A pathologist sectioned the tissue and placed samples into standard tissue embedding cassettes. These tissue samples were then imaged using the HSI system. After imaging, the samples were returned to the pathologist for processing and analysis. The HSI was later compared to the histological analysis. The spectral angle mapping (SAM) and support vector machine (SVM) algorithms were used to classify pixels in the HSI images as healthy or unhealthy in order to delineate margins. Good agreement between margins determined via HSI (using both SAM and SVM) and histology/white light imaging was found.
\end{abstract}

\section{Keywords}

Hyperspectral Imaging, Cancer Detection, Reflectance Spectroscopy, Single 
Pixel Camera

\section{Introduction}

The American Cancer Society estimates that more than 1.8 million people will be diagnosed with cancer and over 600,000 will die from the disease in 2020 [1]. Surgery remains the primary treatment for most solid mass tumors. The objective of surgery is to remove malignant tissue, while minimizing damage to adjacent healthy tissue to preserve function and/or for cosmetic reasons. A significant challenge for cancer surgery is ensuring that no residual malignant tissue is left behind as recurrent tumors lead to high mortality rates [2]. Consequently, the success of cancer surgery depends on a doctor's ability to accurately delineate tumor margins.

Multiple imaging modalities are routinely available for preoperative tumor diagnosis and surgical planning, including $\mathrm{x}$-ray, ultrasound, computed tomography (CT), magnetic resonance imaging (MRI), positron emission tomography (PET) and single photon emission computed tomography (SPECT). However, these techniques are not usually available during surgery. Paraffin section of inked surgical margins is the gold standard for margin assessment. Unfortunately, this process is time-consuming and results are not available until several days after surgery. Typically, surgeons determine the tumor resection margins during procedures based on palpation, visual inspection and frozen section histology. In some cases, intraoperative ultrasound is also used to guide tissue excision [3]. We have constructed a single-pixel hyperspectral imaging (HSI) system to examine the reflectance spectra of tissue. HSI systems are not currently available for bulk tissue analysis during procedures. The long-term goal of this project is to provide an additional intraoperative imaging modality to clearly delineate tumor margins and identify areas of residual disease in real time.

Recent research has demonstrated that optical spectroscopy can be used to distinguish between healthy and diseased or damaged tissue. Both reflectance and fluorescence spectroscopy have been shown to effectively identify unhealthy tissue [4] [5] [6] [7]. Autofluorescence emission is produced by the natural constituents of tissue (no exogenous fluorescent substances are added) when the tissue is illuminated by UV light. The autofluorescence of collagen, near $400 \mathrm{~nm}$, and nicotinamide adenine dinucleotide phosphate, $\mathrm{NAD}(\mathrm{P}) \mathrm{H}$, near $475 \mathrm{~nm}$, have been noted for their high sensitivity to the presence of a range of different diseases [8] [9] [10]. The sensitivity arises because the autofluorescence spectra of proteins change due to alterations in the makeup and structure of unhealthy tissue compared to healthy tissue. Reflectance spectroscopy provides information about tissue morphology. Alterations in tissue morphology, including hyperplasia, nuclear crowding, and increased nuclear/cytoplasmic ratio are associated with disease progression and can cause changes in the wavelength dependence of 
the reflected spectrum [11] [12].

HSI is a hybrid imaging modality that combines imaging and spectroscopy. By collecting spectral information at each position in a 2-D image, HSI generates a 3-D dataset of spatial and spectral information. Because HSI captures both spatial and spectral information, this technique has potential applications for noninvasive disease diagnosis and surgical guidance. Conventional HSI systems employ spatial or spectral scanning to acquire a hypercube. Spatial scanning techniques (pushbroom or whiskbroom systems) obtain a spectrum in one location and then the object/spectrometer is translated to obtain spatial information. The full hyperspectral image is recovered after scanning is complete. In spectral scanning, a 2-D image is projected through a tunable filter or filter wheel and individual images are captured at different wavelengths. The hyperspectral image is reconstructed by combining the separate monochromatic images. Traditional HSI scanning techniques have been successfully applied to a range of diseases including cancer, hemorrhagic shock, heart and retinal diseases [12] [13] [14]. HSI cancer studies have included distinguishing protein biomarkers on individual tumor cells in vitro, analyzing the morphological and structural properties of histological specimens to classify cancer grade, examining the tissue surface to identify precancerous and malignant lesions in vivo, and measuring the tissue blood volume and oxygenation to quantify tumor angiogenesis and metabolism [12].

A challenge with the use of HSI technology for intraoperative imaging has been that traditional scanning techniques suffer from poor temporal and spatial resolution and low optical throughput. Higher spectral and spatial resolution is important as it can potentially capture more subtle spectral and spatial variations of different tissue types necessary for an effective interoperative tool. Low optical throughput hampers the ability to increase spatial and spectral resolution in traditional HSI systems. These problems have led to the development of techniques that can capture the whole data cube in a single snapshot. For example, image mapping spectroscopy (IMS) uses an array of densely packed mirror facets to spatially distribute neighboring image regions to isolated regions on a CCD [15] [16] [17]. A prism then disperses the spectral content from the mapped image into the surrounding empty regions on the CCD. Johnson et al. have used a computed tomographic imaging spectrometer (CTIS) to capture both spatial and spectral information in a single frame for retinal imaging spectroscopy [18]. CTIS captures the spatial and spectral information by imaging the scene through a two-dimensional grating disperser. This produces multiple, spectrally dispersed images that are recorded by a focal plane array.

In this study, the design and testing of a new HSI system based on a single-pixel camera engine are discussed. The primary advantage of a single pixel architecture over traditional scanning HSI techniques is its high sensitivity and potential to function at low light levels. In a single pixel camera, the light gathered from a scene falls onto one pixel. Scanning techniques measures only $1 / \mathrm{N}$ 
of total light per pixel, while CS measures $1 / 2$ of total light in a single detector [19]. This efficient light acquisition of an HSI system based on a single pixel engine provides high sensitivity which can enable the high spatial and spectral resolution needed for intraoperative imaging. Single pixel imaging can provide higher spectral and spatial resolution than other non-scanning HSI techniques such as IMS. While single-pixel HSI is not a snapshot technique, it can in principle acquire images at high rates.

The Nyquist-Shannon sampling theorem states that a band-limited input signal can be recovered without distortion if it is sampled at a rate of at least twice the highest frequency component of interest within the signal. For some signals, such as images that are not naturally band limited, the sampling rate is dictated not by the Nyquist-Shannon theorem but by the desired temporal or spatial resolution. However, it is common in such systems to use an anti-aliasing low-pass filter to band limit the signal before sampling it, and so the Nyquist Shannon theorem plays an implicit role. Recently, an alternative theory, compressive sampling/sensing (CS), has emerged demonstrating that signals can be reconstructed from far fewer measurements than the Nyquist limit [20]. A single pixel camera uses a single detector to create a 2-D image of a scene rather than using a traditional array detector and relies on the mathematical theory and algorithms of CS. CS relies on the empirical observation that many types of signals or images can be well approximated by a sparse expansion in terms of a suitable basis, that is, by only a small number of non-zero coefficients. This is the key aspect of many lossy compression techniques such as JPEG and MP3, where compression is achieved by storing only the largest basis coefficients. In mathematical form, a signal, $X$, can be represented in some basis, $\Psi$, such that

$$
x=\sum_{i=1}^{N} a_{i} \Psi_{i}
$$

If only a small number, $m$, of the coefficients, $a_{i}$, are nonzero then $x$ is $m$-sparse in the domain $\Psi$. In addition, the signal $x$ is compressible if the representation in Equation (1) has just a few large coefficients.

In conventional imaging systems, an image is captured and then compressed. CS offers the opportunity to capture the image directly in its compressed form. Instead of measuring an $N$-pixel sampled version of $x$, the incident light-field from a scene, CS acquires a condensed representation of $M<N$ linear measurements of $x$ and a set of test functions $\left\{\varphi_{m}\right\}_{m=1}^{M}$ such that [21]

$$
y=\Phi x
$$

Incoherence requires that the rows of $\Phi$ (the measurement matrix) cannot represent the columns of $\Psi$ in a sparse way (and vice-versa). Random matrices are largely incoherent with any fixed basis $\Psi$. This allows the use of known fast transforms such as a Walsh, Hadamard, or Noiselet transforms as the test function for CS [22].

In CS, the number of samples taken is smaller than the number of coefficients in the full image or signal. Converting the information back to the intended do- 
main involves solving an underdetermined matrix equation meaning there could be a large number of candidate solutions. Consequently, a strategy to select the best solution is needed. Different approaches to recover information from incomplete data sets have existed for several decades. Candès et al. [23], discovered that this reconstruction problem could be seen as an optimization problem and be efficiently solved using the $\ell_{1}$-norm. Their discoveries made it possible to reconstruct MRI data from what appeared to be highly incomplete data sets in terms of the Nyquist-Shannon criterion.

One of the first realizations of a single-pixel imaging system based on the idea of CS was developed at Rice University [24]. In this system, light reflected from an object was multiplied by a random code and then captured by a single detector. The random code multiplication was accomplished by a spatial light modulator. The signal samples from detector are then processed to reconstruct the image. Other researchers have developed compressive spectral imaging systems based on the single-pixel-camera design, [25] [26] coded apertures [27] [28] [29] and schemes based on spectral modulation [30]. However, they do not offer sufficient wavelength resolution or the dynamic range needed to be an effective medical imaging technique. The single pixel imaging system described here overcomes these issues.

The single-pixel camera engine used in the HSI described here uses a digital micromirror device (DMD) as a spatial light modulator to optically calculate linear projections of a scene onto pseudo-random binary patterns. Hadamard matrices, which fulfill the sparsity requirements for CS, are used as the binary patterns. A single-pixel imaging system produces an image by rapidly obtaining many measurements of the intensity of a scene using different Hadamard matrices. Typically, a single-pixel imaging system functions in one wavelength band. In the HSI system described here, this method of single-pixel imaging based on CS has been made to be hyperspectral by replacing the single detector with a spectrometer. The dispersive element of the spectrometer spreads the light across the linear detector array. Each pixel along the array acts as a single pixel detector independently operating at a specific wavelength. The reconstruction of an image can be performed using any of the pixels, which corresponds to a reconstruction as a function of wavelength, or HSI [31] [32].

In this pilot study, our HSI system based on a single-pixel camera design is used to image human tissue for the first time to access the applicability of this single pixel approach to cancer detection. This paper presents the results of a study that included 19 patients to assesses the ability of the HSI system to use reflectance imaging to delineate adenocarcinoma tumor margins in human pancreatic tissue imaged ex vivo. Hyperspectral images were analyzed and margins were identified using the Spectral Angle Mapping (SAM) algorithm [33]. In addition, the support vector machine (SVM) algorithm [34] was used to analyze a subset of samples and these results were compared to the SAM analysis. The HSI results were compared to margins determined via histology and white light imaging. 


\section{Methods}

Pancreatic tissue excised during pancreatectomy was imaged immediately after being sent to the pathology lab, before processing or staining. After receiving the sample, a pathologist sectioned the tissue into $2 \mathrm{~cm} \times 2 \mathrm{~cm} \times 0.5 \mathrm{~cm}$ pieces and placed samples into standard tissue embedding cassettes. These tissue samples were then imaged using the HSI system. After imaging, the samples were returned to the pathologist for processing and analysis. The HSI was later compared to the histological analysis. Tissue samples were divided into three groups: malignant tissue, healthy tissue, or tissue containing both malignant and healthy tissue (a margin). Some patients contributed tissue samples to multiple groups. The number of tissue samples in each group is shown in Table 1.

Figure 1 shows a schematic of the HSI system. This setup is similar to the single pixel camera described by [24] with the single detector replaced by a spectrometer to enable HSI. A white light source illuminates the tissue sample, and an image of the sample is formed on the DMD by the means of a lens. The DMD serves as the spatial light modulator in the system and is programmed to multiply the light with the test functions. The spatially modulated light is focused to a point by a collection lens and launched into a fiber to a spectrometer. In the

Table 1. Number and type of pancreatic tissue samples used in study.

\begin{tabular}{cccc}
\hline \multirow{2}{*}{$\begin{array}{c}\text { Number and Type } \\
\text { of Pancreatic Tissue }\end{array}$} & Healthy & Malignant & Margin \\
\cline { 2 - 4 } & 12 & 9 & 19 \\
\hline
\end{tabular}

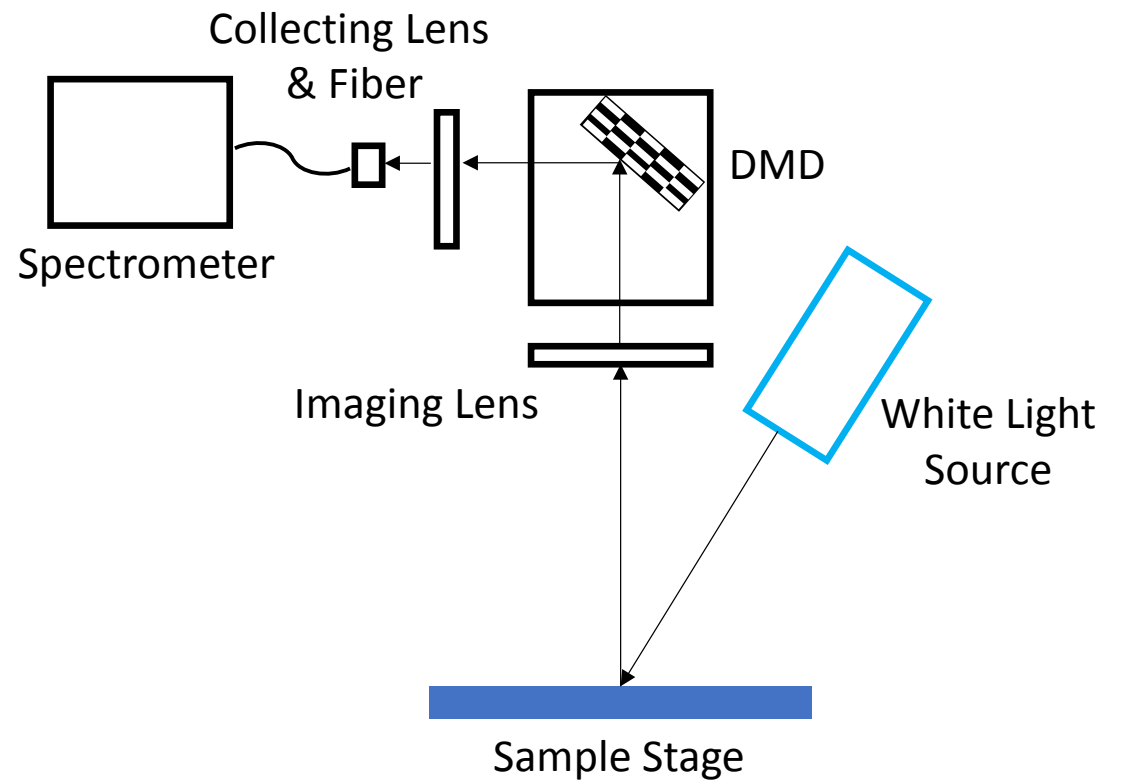

Figure 1. Schematic of the hyperspectral imaging system. The sample stage is illuminated by a white light source. Light is collected from the sample by an imaging optic and an image is formed on the DMD. The light is then reflected off the DMD through a series of collection optics and into a fiber where the signal is sent to a spectrometer. 
spectrometer, light is dispersed and directed to a linear array detector. The signal at each pixel of the array detector corresponds to a particular wavelength. Each of the pixels along the array functions as a single pixel camera, each at a different wavelength. The digital micromirror device (DMD) in the system is a DLP5500 0.55 " XGA with $1024 \times 768$ pixels. The spectrometer is an Ocean Optics QE Pro Spectrometer (spectral range $350-750 \mathrm{~nm}, 1044$ pixels in the linear array, and dispersion $0.335 \mathrm{~nm} /$ pixel). The spatial resolution of the system is set by the size of the pixels used in the binary codes used on the DMD. In this study, $32 \times 32$ Hadamard matrices were used on the DMD, with a FOV of $1.9 \mathrm{~cm} \times 1.9 \mathrm{~cm}$, resulting in a spatial resolution of $0.44 \mathrm{~mm} /$ pixel. LabVIEW software was used to manipulate the DMD and capture the signal from the spectrometer.

The HSI imaging system was mounted on a vertical stage and imaged the tissue samples from above (see Figure 1). Either a halogen lamp (150 W, Model I-150 Optical Fiber Light Source, Cuda) or xenon (300 W, Circon MV 9086) source was used as the source for reflectance spectroscopy. Both light sources were mounted $23^{\circ}$ from the vertical axis and aligned to illuminate the specimen. This provided uniform illumination across the $1.9 \mathrm{~cm} \times 1.9 \mathrm{~cm}$ imaging area.

To begin the experiment, a series of Hadamard matrices were generated, each a matrix of zeros and ones (these were the test functions for CS). These pseudorandom codes were loaded into the DMD and applied one at a time. The mirrors whose pixels were designated as zero in the code were rotated to reflect light away from the spectrometer detector array, while mirrors with pixels designated as one were rotated to reflect light onto the detector array. The use of pseudorandom measurement patterns, linked to the programmable mirror positions in the DMD, ensured that the light signal was compressible and universally incoherent. For each code, the output signal from each pixel along the linear array of the detector was stored in a vector with its location index corresponding to its respective code. To obtain reflectance imaging, 800 Hadamard patterns were used on the DMD with an integration time of $100 \mathrm{~ms}$ for each code to acquire the intensity measurements needed to reconstruct the image. Total acquisition time was approximately 7 minutes. After image acquisition a MATLAB program based on the NESTA algorithm was used to reconstruct the compressed images [35]. NESTA is a fast and robust first-order method than solves basis-pursuit problems.

In addition to the hyperspectral imaging, a white light image was obtained for all samples using a traditional 8-megapixel CMOS array camera (iSight camera). These white light images do not contain spectral information. The morphology of structures seen in these white light images was compared to structures seen in the hyperspectral images and in the histology slides.

\section{Results and Discussion}

\subsection{Spectral Angle Mapping}

SAM analysis compares a known reference spectrum to the spectrum of each 
pixel in a spectral image of an object to look for differences between the two [33]. SAM has been used by other researchers for the classification of HSI including the identification of nerve fibers [36] and for the automatic detection of altered mucosa of the human larynx [37]. To create the reference spectrum, two wavelength bands of interest are chosen and the intensities of all pixels in the reference image are determined in these two bands. These pixel intensities are graphed as a scatterplot and a linear regression is performed to define a reference vector. For comparison, a vector is created for each pixel in the image of the object. For each pixel, the intensity of the pixel in the two wavelength bands is plotted and the vector is drawn from the origin to this point. The reference and pixel vectors are then compared using the normalized dot product producing a spectral angle between the two vectors. This method is insensitive to differences in intensity since the SAM algorithm uses only the vector direction and not the vector magnitude for comparison. In this study, this process was modified so that it was repeated for multiple wavelengths to create a hyperspectral angle.

Figure 2 shows the reflectance spectra of a pancreatic tissue and illustrates the differences between healthy and cancerous tissue. Absorption by blood (near 575 $\mathrm{nm}$ ) is evident in the spectra. A series of reference vectors was created using the hyperspectral images of healthy pancreatic tissue for reflectance imaging. There was significant variation in the spectra of both healthy and malignant tissue from patient to patient. Because of this, reference vectors were determined on a patient-by-patient basis. The shape of the reflectance spectra of healthy and cancerous tissue are similar between 650 and $675 \mathrm{~nm}$, but large differences are apparent from 450 to $520 \mathrm{~nm}$. The $\mathrm{x}$-coordinate for all reference vectors for reflectance imaging was the average intensity over the $650-655 \mathrm{~nm}$ wavelength band in the image of healthy tissue. The wavelength band used for the y-coordinate for the reference vectors was varied in $5 \mathrm{~nm}$ increments across the wavelength

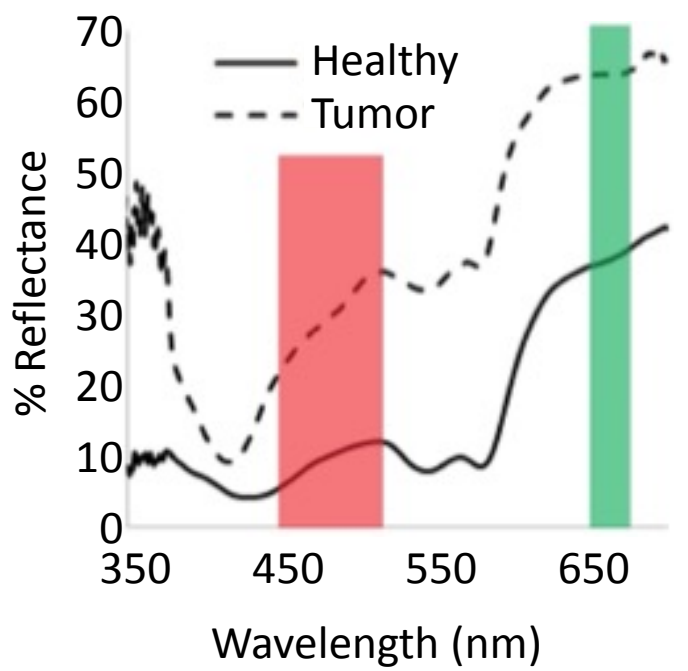

Figure 2. The reflectance spectrum of healthy and cancerous pancreatic tissue. The red and green regions indicate the wavelength ranges used to construct vectors for SAM analysis. 
band $450-520 \mathrm{~nm}$. This analysis resulted in a series of reference vectors across multiple wavelengths for each tissue sample.

\subsection{SAM Analysis of Reflectance Images of Healthy Pancreatic Tissue}

Samples of healthy pancreatic tissue were obtained from 12 patients. These tissue samples did not contain any malignant tissue. We did not obtain samples of healthy tissue from all patients because in some cases regions of healthy tissue could not be identified by the pathologist due to the extent of disease. SAM analysis was applied to these images of healthy tissue to provide a measure of how uniform the spectra of the healthy tissue were as a function of position across the sample. A series of spectral angles was calculated for each reference vector and these spectral angles were summed to form a hyperspectral angle. Figure 3 shows representative examples of white light and HSI of healthy pancreatic tissue. The white light images have been scaled so that they have the same spatial resolution as the HSI. Some structures within the tissue are evident in the white light image. However, these structures are not seen in the HSI indicating that they have the same spectrum as healthy tissue. For the healthy tissue the spectral angle is approximately constant across the sample. The hyperspectral angle has an average value of $0.98^{\circ} \pm 4.02^{\circ}$ for the reflectance images of the 12 samples. This small angle indicates good agreement between the reference spectra and the spectra in the pixels in each of the hyperspectral images of healthy tissue.

\subsection{SAM Analysis of Reflectance Images of Malignant Pancreatic Tissue}

Samples of entirely malignant pancreatic tissue were obtained for 9 patients. SAM analysis was applied to the images of these samples to access the ability of HSI to identify malignant tissue. In all cases, a series of reference vectors of healthy tissue were formed from a sample of healthy tissue for each patient. SAM analysis was then performed on the images of malignant tissue comparing the spectra in these images to the healthy tissue reference vectors. Figure 4 shows representative examples of white light and HSI of malignant pancreatic tissue.
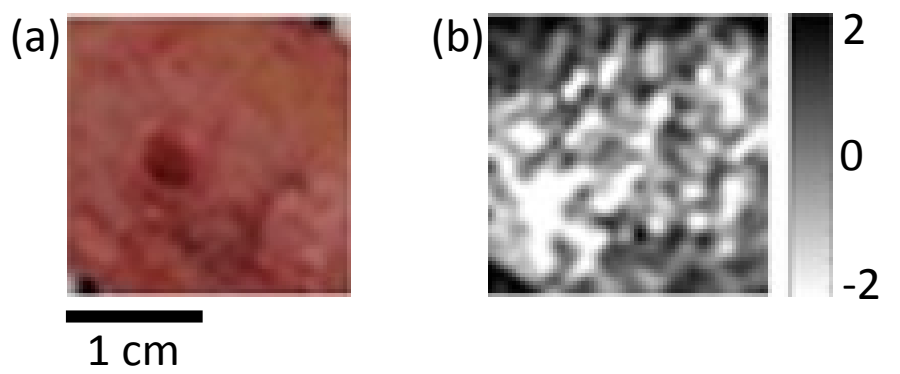

Figure 3. (a) White light image of healthy pancreatic tissue. The red circular structure near the center of the image is a blood vessel. (b) The SAM image based on the HSI. The spectral angle is approximately uniform across the tissue. 
(a)

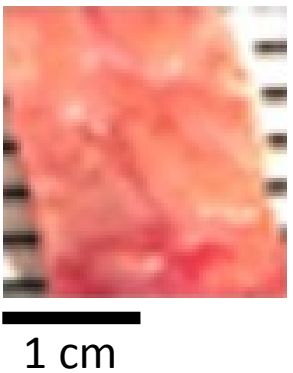

(b)

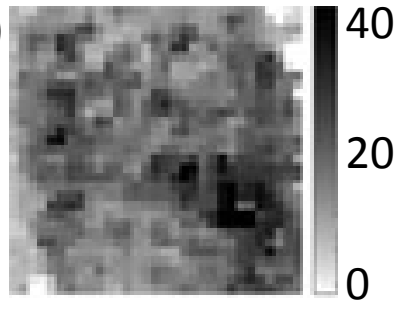

Figure 4. (a) White light image of a tissue sample that was entirely malignant. (b) The reconstructed SAM image.

The spectral angle has an average value of $35.84^{\circ} \pm 1.34^{\circ}$ for the 9 tissue samples. This large spectral angle indicates that the spectrum of malignant tissue is significantly different from healthy tissue. HSI imaging and SAM analysis are capable of differentiating between healthy and malignant tissue.

\subsection{SAM Analysis of Reflectance Images of Samples Containing both Healthy and Malignant Pancreatic Tissue}

Tissue samples from 19 patients contained transition regions from healthy to cancerous tissue-margins. SAM analysis was applied to these images, using the spectrum of healthy tissue for each patient as the reference vector. Figure 5 shows representative examples of white light and HSI of a sample containing both healthy and malignant tissue. In the HSI, regions with large spectral angles are regions in which the spectrum of the tissue is significantly different from that of healthy tissue. Regions in the HSI where the hyperspectral angle was two standard deviations away from the reference vector for healthy tissue were identified as malignant. The HSI results were compared to histology. The total number of pixels classified in the HSI as malignant was determined for each tissue sample. The histology slide images were overlaid with the white light images and malignant regions were identified in the white light images. These white light images were scaled to the same spatial resolution as the HSI and the total number of pixels classified as malignant was determined for each sample. These pixel areas as measured via SAM and in white light/histology were compared using a Bland-Altman plot [38]. Figure 6 shows good agreement between lesion areas as measured in the reflectance HSI via SAM and white light/histology images. All measurements fall within two standard deviations of the mean difference and there is little difference between the bias and the line of equality. This plot suggests that the HSI in combination with SAM is imaging the same cancerous regions as is seen in the histology slides. The HSI system is sensitive to the spectral signature of cancer in the tissue.

\subsection{SVM Analysis of Reflectance Images of Samples Containing both Healthy and Malignant Pancreatic Tissue}

SVM is a kernel-based machine learning technique that has been widely used in 
(a)

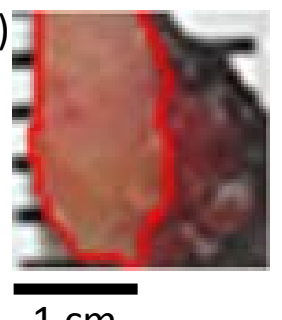

(b)

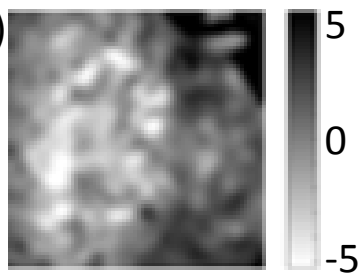

(c)

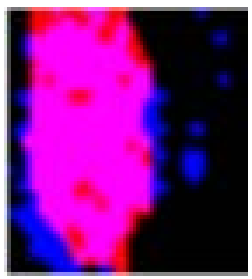

Figure 5. (a) A white light image of pancreatic tissue. The red line denotes the border of the malignant region as identified by histological analysis. (b) The reconstructed SAM image, the lighter color corresponds to a larger spectral angle indicting the presence of malignant tissue. (c) A comparison of the pixels identified as malignant via HSI and via histology. The red pixels correspond to the pixels identified as malignant via histology, the blue pixels were identified by HSI and pink/purple pixels were identified using both techniques.

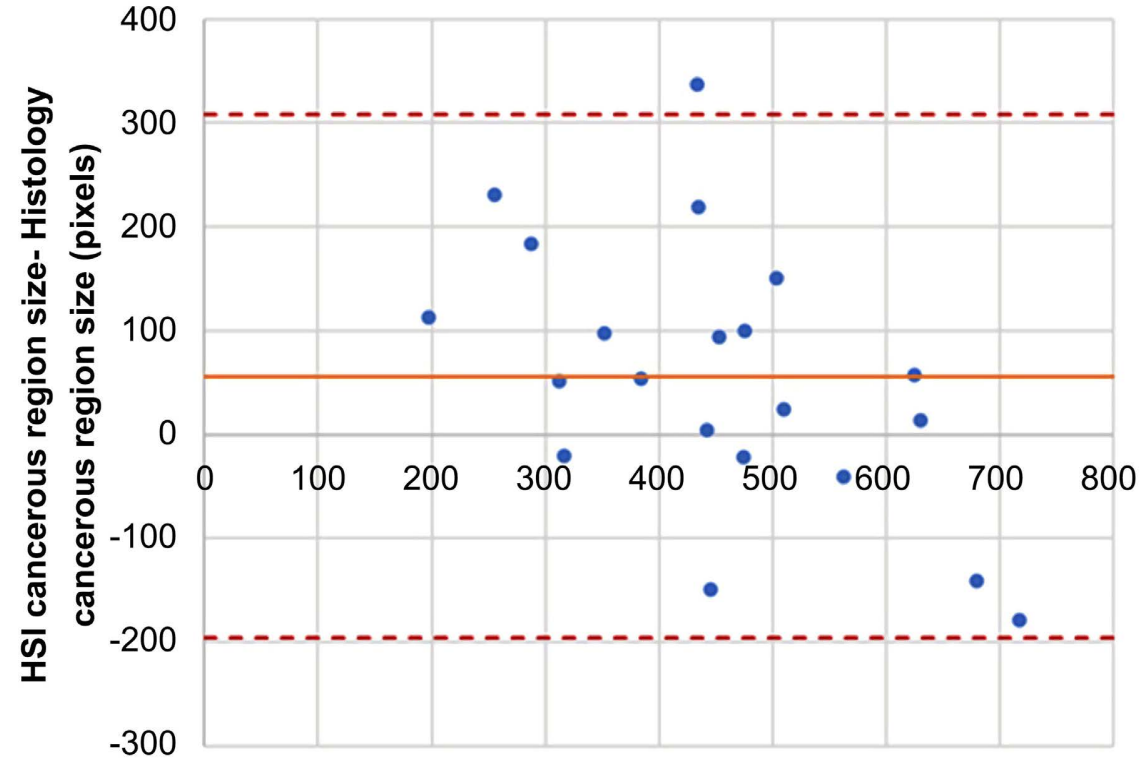

Average Cancerous Region size (pixels)

Figure 6. A Bland-Altman plot comparing the areas of the malignant regions as determined in the HSI using SAM and white light/histological images of pancreatic tissue. The solid orange line represents the mean of the difference, and the dotted red lines show two standard deviations of the mean difference. Most of the measurements fall within two standard deviations of the mean difference and there is little difference between the bias and the line of equality.

the classification of hyperspectral images [39] [40] [41] [42]. For comparison, SVM analysis of a subset of 10 tissue samples from the 19 that contained transition regions from healthy to cancerous tissue was performed. In each image, eight pixels were selected to build the training model for SVM. Four of these pixels were labeled as healthy, and the other four pixels were labeled as unhealthy. The unhealthy pixels were given a value of 1 and the resulting classified pixel values were summed together to give an overall count for the number of unhealthy pixels. These values were then compared to the number of pixels classi- 
fied by SAM and white light histology. The pixel areas as measured via SVM and in the white light/histology were compared using a Bland-Altman plot (see Figure 7). This graph shows good agreement between lesion areas as measured in the reflectance HSI via SVM and white light/histology images. All measurements fall within two standard deviations of the mean difference and there is little difference between the bias and the line of equality. Figure 8 displays a Bland-Altman

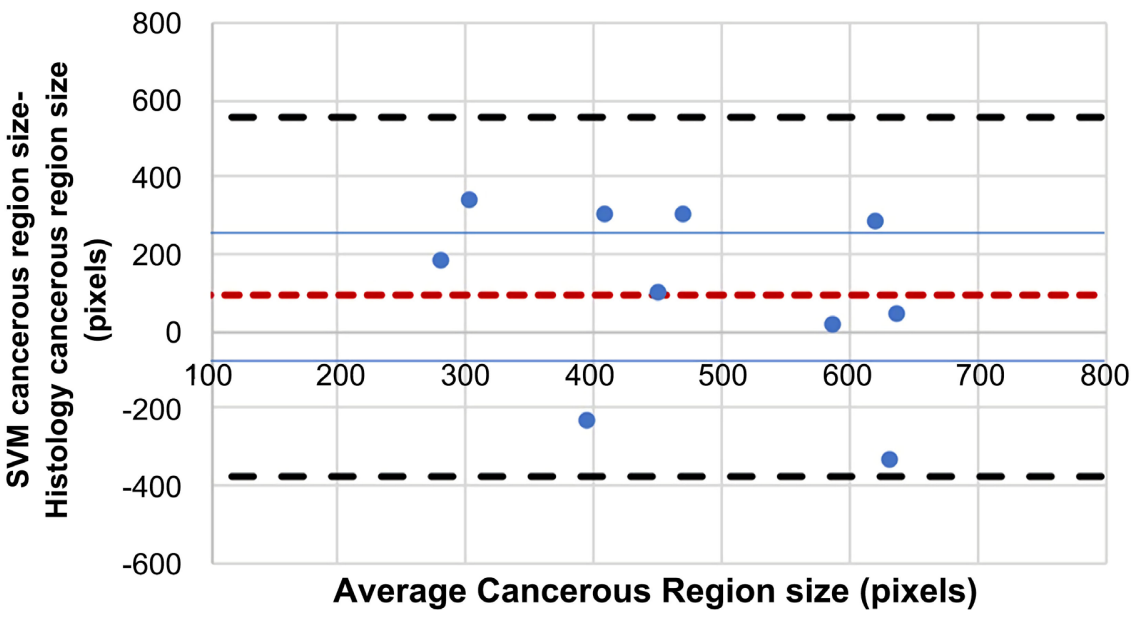

Figure 7. A Bland-Altman plot comparing the areas of the malignant regions as determined in the HIS using SVM and white light/histological images of pancreatic tissue. The dotted red line represents the mean of the difference. The dotted black lines show two standard deviations of the mean difference, and the thin blue lines show the limits of the $95 \%$ confidence interval. Most of the measurements fall within two standard deviations of the mean difference and there is little difference between the bias and the line of equality.

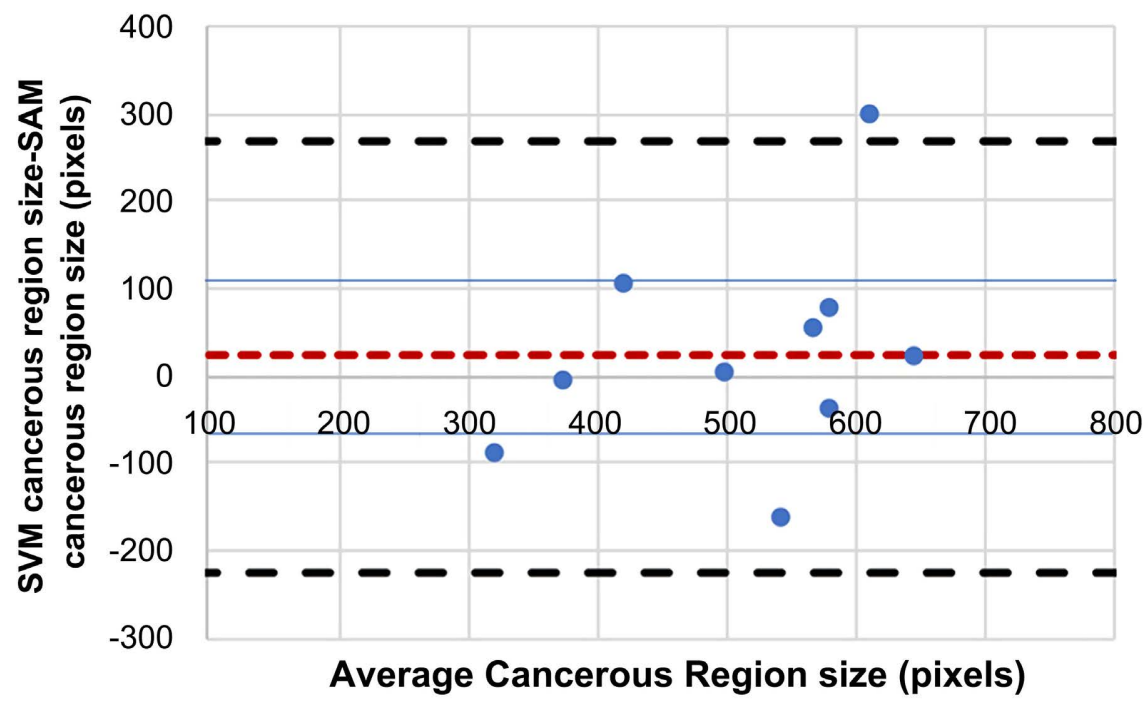

Figure 8. A Bland-Altman plot comparing the areas of the malignant regions as determined in the HIS using SVM versus SAM. The dotted red line represents the mean of the difference. The dotted black lines show two standard deviations of the mean difference, and the thin blue lines show the limits of the $95 \%$ confidence interval. Most of the measurements fall within two standard deviations of the mean difference and there is little difference between the bias and the line of equality. 
plot comparing the classification method based upon SVM versus SAM. The majority of points (all but one) fall within the limits of agreement and there is no apparent trend showing a bias towards one method or another. The two classification methods seem to work equally well at classifying the HSI data in this study.

\section{Conclusions}

An HSI system based on a single-pixel camera design for use in discriminating between healthy and malignant pancreatic tissue based on the reflectance spectra of the tissue has been constructed and tested. Pancreatic tissue samples containing a margin from 19 patients were imaged ex vivo using the single-pixel HSI system in reflectance mode. Good agreement was found between the areas of the cancerous regions measured in the reflectance HSI and the areas measured via white light imaging augmented with histology using both SAM and SVM classification algorithms. These results confirm that our camera is sensitive to spectral differences between healthy and malignant pancreatic tissue. The system currently takes about 7 minutes to collect a single image which is insufficient for "real-time" imaging. The current limitation on acquisition time is hardware (both the speed of the DMD and computer) that can be overcome in future imaging systems. Further, other groups have shown recently that single pixel compressive imaging can be made faster [43]. In addition, for this technology to be most useful in an intraoperative setting, we will integrate HSI imaging into endoscopic and laparoscopic probes using a coherent fiber bundle. Ekpenyong pointed out that it can be difficult to calibrate HIS systems at low light levels [44]. The single-pixel system described here is designed to function efficiently at low light levels and may help alleviate these difficulties.

Research has shown that autofluorescence spectroscopy of tissue can be used in conjunction with reflectance spectroscopy to improve the specificity and sensitivity of cancerous regions in tissue [45]. Adding the ability to image autofluorescence spectra in future studies will improve our detection technique. The autofluorescence signal of collagen and $\mathrm{NAD}(\mathrm{P}) \mathrm{H}$ are very faint and had a lower
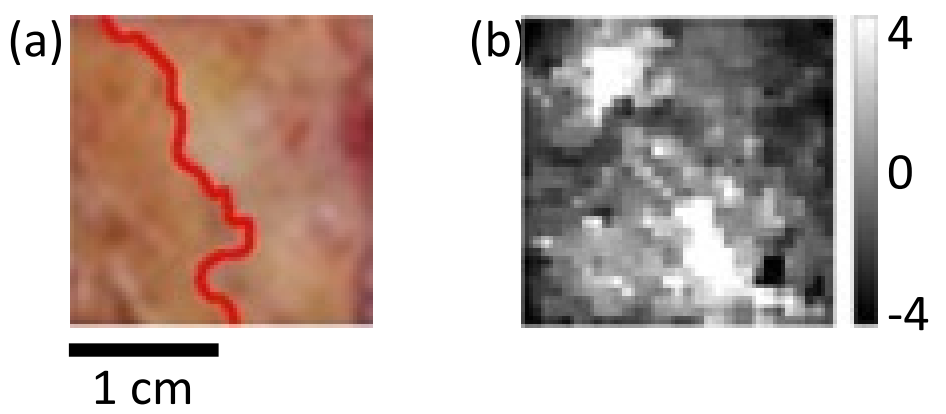

Figure 9. (a) A white light image of a pancreatic tissue sample. The red line denotes the border of the malignant region. In this case, all tissue on the right of this line was classified as malignant via histology. (b) The reconstructed SAM image, the lighter color corresponds to a large spectral angle relative to the reference vector for healthy tissue. 
signal to noise than the reflectance images. For this reason, the high sensitivity of single pixel imaging makes it preferable to other imaging modalities. We have performed a pilot study of HSI autofluorescence imaging on pancreatic tissue. A $3 \mathrm{~mW}$ nitrogen laser (SRS, NL100) was used as the excitation source for autofluorescence spectroscopy. Preliminary results for one patient in this study are shown in Figure 9. We obtained HSI of a sample of healthy tissue, and a margin sample for this patent and performed SAM analysis of these images as outlined in Section 3.1. Figure 9 shows the white light and HSI of the margin sample, the boundary between the malignant tissue and the healthy tissue is shown in red. In the HSI, regions with large spectral angles are regions in which the spectrum of the tissue is significantly different from that of healthy tissue.

Before this HSI technique can be used in a surgical setting, the effects of blood profusion must be addressed. In the study presented here, no blood was present on the surface of the tissue; however, many groups have shown the presence of blood absorption affects the spectroscopy of tissue [46]. We will need to next test this technique on a live animal model to investigate these effects in more detail.

\section{Funding}

This work was supported by a grant from the Levine Cancer Institute, Charlotte, NC.

\section{Acknowledgements}

The authors would like to thank the staff of the Pathology Lab at Carolinas Medical Center for their assistance in preparing the tissue samples for imaging.

\section{Conflicts of Interest}

The authors have no relevant financial interests in the manuscript and no other potential conflicts of interest to disclose.

\section{References}

[1] Siegel, R.L., Miller, K.D. and Jemal, A. (2020) Cancer Statistics, 2020. CA: A Cancer Journal for Clinicians, 70, 7-30. https://doi.org/10.3322/caac.21590

[2] Gage, I., Schnitt, S.J., Nixon, A.J., Silver, B., Recht, A., Troyan, S.L., Eberlein, T., Love, S.M., Gelman, R. and Harris, J.R. (1996) Pathologic Margin Involvement and the Risk of Recurrence in Patients Treated with Breast-Conserving Therapy. Cancer. Interdisciplinary International Journal of the American Cancer Society, 78, 1921-1928.

https://doi.org/10.1002/(SICI)1097-0142(19961101)78:9<1921::AID-CNCR12>3.0.C $\mathrm{O} ; 2-\#$

[3] Cleary, K. and Peters, T.M. (2010) Image-Guided Interventions: Technology Review and Clinical Applications. Annual Review of Biomedical Engineering, 12, 119-142. https://doi.org/10.1146/annurev-bioeng-070909-105249

[4] Volynskaya, Z.I., Haka, A.S., Bechtel, K.L., Fitzmaurice, M., Shenk, R., Wang, N., Nazemi, J., Dasari, R.R. and Feld, M.S. (2008) Diagnosing Breast Cancer Using Diffuse Reflectance Spectroscopy and Intrinsic Fluorescence Spectroscopy. Journal of 
Biomedical Optics, 13, Article ID: 024012. https://doi.org/10.1117/1.2909672

[5] Zonios, G., Perelman, L.T., Backman, V., Manoharan, R., Fitzmaurice, M., Van Dam, J. and Feld, M.S. (1999) Diffuse Reflectance Spectroscopy of Human Adenomatous Colon Polyps in Vivo. Applied Optics, 38, 6628-6637. https://doi.org/10.1364/AO.38.006628

[6] Chang, S.K., Marín, N., Follen, M. and Richards-Kortum, R.R. (2006) Model-Based Analysis of Clinical Fluorescence Spectroscopy for in Vivo Detection of Cervical Intraepithelial Dysplasia. Journal of Biomedical Optics, 11, Article ID: 024008. https://doi.org/10.1117/1.2187979

[7] Georgakoudi, I. and Feld, M.S. (2004) The Combined Use of Fluorescence, Reflectance, and Light-Scattering Spectroscopy for Evaluating Dysplasia in Barrett's Esophagus. Gastrointestinal Endoscopy Clinics, 14, 519-537.

https://doi.org/10.1016/j.giec.2004.03.008

[8] Ramanujam, N., Mitchell, M.F., Mahadevan, A., Warren, S., Thomsen, S., Silva, E. and Richards-Kortum, R. (1994) In Vivo Diagnosis of Cervical Intraepithelial Neoplasia Using 337-nm-Excited Laser-Induced Fluorescence. Proceedings of the National Academy of Sciences, 91, 10193-10197.

https://doi.org/10.1073/pnas.91.21.10193

[9] Cothren, R., Richards-Kortum, R., Sivak Jr., M., Fitzmaurice, M., Rava, R., Boyce, G., Doxtader, M., Blackman, R., Ivanc, T. and Hayes, G. (1990) Gastrointestinal Tissue Diagnosis by Laser-Induced Fluorescence Spectroscopy at Endoscopy. Gastrointestinal Endoscopy, 36, 105-111. https://doi.org/10.1016/S0016-5107(90)70961-3

[10] Schomacker, K.T., Frisoli, J.K., Compton, C.C., Flotte, T.J., Richter, J.M., Nishioka, N.S. and Deutsch, T.F. (1992) Ultraviolet Laser-Induced Fluorescence of Colonic Tissue: Basic Biology and Diagnostic Potential. Lasers in Surgery and Medicine, 12, 63-78. https://doi.org/10.1002/lsm.1900120111

[11] Wallace, M.B., Perelman, L.T., Backman, V., Crawford, J.M., Fitzmaurice, M., Seiler, M., Badizadegan, K., Shields, S.J., Itzkan, I. and Dasari, R.R. (2000) Endoscopic Detection of Dysplasia in Patients with Barrett's Esophagus Using Light-Scattering Spectroscopy. Gastroenterology, 119, 677-682. https://doi.org/10.1053/gast.2000.16511

[12] Lu, G. and Fei, B. (2014) Medical Hyperspectral Imaging: A Review. Journal of Biomedical Optics, 19, Article ID: 010901. https://doi.org/10.1117/1.JBO.19.1.010901

[13] Lu, G., Halig, L.V., Wang, D., Qin, X., Chen, Z.G. and Fei, B. (2014) Spectral-Spatial Classification for Noninvasive Cancer Detection Using Hyperspectral Imaging. Journal of Biomedical Optics, 19, Article ID: 106004.

https://doi.org/10.1117/1.JBO.19.10.106004

[14] Cancio, L.C., Batchinsky, A.I., Mansfield, J.R., Panasyuk, S., Hetz, K., Martini, D., Jordan, B.S., Tracey, B. and Freeman, J.E. (2006) Hyperspectral Imaging: A New Approach to the Diagnosis of Hemorrhagic Shock. Journal of Trauma and Acute Care Surgery, 60, 1087-1095. https://doi.org/10.1097/01.ta.0000217357.10617.3d

[15] Kester, R.T., Bedard, N., Gao, L.S. and Tkaczyk, T.S. (2011) Real-Time Snapshot Hyperspectral Imaging Endoscope. Journal of Biomedical Optics, 16, Article ID: 056005. https://doi.org/10.1117/1.3574756

[16] Elliott, A.D., Gao, L., Ustione, A., Bedard, N., Kester, R., Piston, D.W. and Tkaczyk, T.S. (2012) Real-Time Hyperspectral Fluorescence Imaging of Pancreatic $\beta$-Cell Dynamics with the Image Mapping Spectrometer. Journal of Cell Science, 125, 
4833-4840. https://doi.org/10.1242/jcs. 108258

[17] Fawzi, A.A., Lee, N., Laine, A.F., Acton, J.H. and Smith, R.T. (2011) Recovery of Macular Pigment Spectrum in Vivo Using Hyperspectral Image Analysis. Journal of Biomedical Optics, 16, Article ID: 106008. https://doi.org/10.1117/1.3640813

[18] Johnson, W.R., Wilson, D.W., Fink, W., Humayun, M.S. and Bearman, G.H. (2007) Snapshot Hyperspectral Imaging in Ophthalmology. Journal of Biomedical Optics, 12, Article ID: 014036. https://doi.org/10.1117/1.2434950

[19] Baraniuk, R.G. (2007) Compressive Sensing [Lecture Notes]. IEEE Signal Processing Magazine, 24, 118-121. https://doi.org/10.1109/MSP.2007.4286571

[20] Candès, E.J. and Wakin, M.B. (2008) An Introduction to Compressive Sampling. IEEE Signal Processing Magazine, 25, 21-30. https://doi.org/10.1109/MSP.2007.914731

[21] Duarte, M.F., Davenport, M.A., Takhar, D., Laska, J.N., Sun, T., Kelly, K.F. and Baraniuk, R.G. (2008) Single-Pixel Imaging via Compressive Sampling. IEEE Signal Processing Magazine, 25, 83-91. https://doi.org/10.1109/MSP.2007.914730

[22] Candès, E.J. and Romberg, J. (2007) Sparsity and Incoherence in Compressive Sampling. Inverse Problems, 23, 969-985. https://doi.org/10.1088/0266-5611/23/3/008

[23] Candès, E.J., Romberg, J. and Tao, T. (2006) Robust Uncertainty Principles: Exact Signal Reconstruction from Highly Incomplete Frequency Information. IEEE Transactions on Information Theory, 52, 489-509. https://doi.org/10.1109/TIT.2005.862083

[24] Takhar, D., Laska, J.N., Wakin, M.B., Duarte, M.F., Baron, D., Sarvotham, S., Kelly, K.F. and Baraniuk, R.G. (2006) A New Compressive Imaging Camera Architecture Using Optical-Domain Compression. Proceedings of Computational Imaging $V$, Vol. 6065, 606509. https://doi.org/10.1117/12.659602

[25] August, Y., Vachman, C., Rivenson, Y. and Stern, A. (2013) Compressive Hyperspectral Imaging by Random Separable Projections in Both the Spatial and the Spectral Domains. Applied Optics, 52, D46-D54. https://doi.org/10.1364/AO.52.000D46

[26] Lin, X., Wetzstein, G., Liu, Y. and Dai, Q. (2014) Dual-Coded Compressive Hyperspectral Imaging. Optics Letters, 39, 2044-2047. https://doi.org/10.1364/OL.39.002044

[27] Gehm, M.E., John, R., Brady, D.J., Willett, R.M. and Schulz, T.J. (2007) Single-Shot Compressive Spectral Imaging with a Dual-Disperser Architecture. Optics Express, 15, 14013-14027. https://doi.org/10.1364/OE.15.014013

[28] Golub, M.A., Averbuch, A., Nathan, M., Zheludev, V.A., Hauser, J., Gurevitch, S., Malinsky, R. and Kagan, A. (2016) Compressed Sensing Snapshot Spectral Imaging by a Regular Digital Camera with an Added Optical Diffuser. Applied Optics, 55, 432-443. https://doi.org/10.1364/AO.55.000432

[29] Arce, G.R., Brady, D.J., Carin, L., Arguello, H. and Kittle, D.S. (2013) Compressive Coded Aperture Spectral Imaging: An Introduction. IEEE Signal Processing Magazine, 31, 105-115. https://doi.org/10.1109/MSP.2013.2278763

[30] August, I., Oiknine, Y., AbuLeil, M., Abdulhalim, I. and Stern, A. (2016) Miniature Compressive Ultra-Spectral Imaging System Utilizing a Single Liquid Crystal Phase Retarder. Scientific Reports, 6, Article No. 23524. https://doi.org/10.1038/srep23524

[31] Peller, J., Farahi, F. and Trammell, S. (2018) Hyperspectral Imaging System Based on a Single-Pixel Camera Design for Detecting Differences in Tissue Properties. Applied Optics, 57, 7651-7658. https://doi.org/10.1364/AO.57.007651 
[32] Abolbashari, M., Farahi, F., Magalhaes, F., Araújo, F.M. and Correia, M.V. (2012) High Dynamic Range Compressive Imaging: A Programmable Imaging System. Optical Engineering, 51, Article ID: 071407. https://doi.org/10.1117/1.OE.51.7.071407

[33] Kruse, F.A., Lefkoff, A., Boardman, J., Heidebrecht, K., Shapiro, A., Barloon, P. and Goetz, A. (1993) The Spectral Image Processing System (SIPS)-Interactive Visualization and Analysis of Imaging Spectrometer Data. Remote Sensing of Environment, 44, 145-163. https://doi.org/10.1016/0034-4257(93)90013-N

[34] Cristianini, N. and Shawe-Taylor, J. (2000) An Introduction to Support Vector Machines and Other Kernel-Based Learning Methods. Cambridge University Press, Cambridge. https://doi.org/10.1017/CBO9780511801389

[35] Becker, S., Bobin, J. and Candès, E.J. (2011) NESTA: A Fast and Accurate First-Order Method for Sparse Recovery. SIAM Journal on Imaging Sciences, 4, 1-39. https://doi.org/10.1137/090756855

[36] Li, Q., Wang, Y., Liu, H. and Chen, Z. (2012) Nerve Fibers Identification Based on Molecular Hyperspectral Imaging Technology. IEEE International Conference on Computer Science and Automation Engineering, Seoul, 20-24 August 2012, 15-17. https://doi.org/10.1109/CSAE.2012.6272897

[37] Martin, R., Thies, B. and Gerstner, A.O. (2012) Hyperspectral Hybrid Method Classification for Detecting Altered Mucosa of the Human Larynx. International Journal of Health Geographics, 11, Article No. 21. https://doi.org/10.1186/1476-072X-11-21

[38] Bland, J.M. and Altman, D. (1986) Statistical Methods for Assessing Agreement between Two Methods of Clinical Measurement. The Lancet, 327, 307-310. https://doi.org/10.1016/S0140-6736(86)90837-8

[39] Akbari, H., Uto, K., Kosugi, Y., Kojima, K. and Tanaka, N. (2011) Cancer Detection Using Infrared Hyperspectral Imaging. Cancer Science, 102, 852-857. https://doi.org/10.1111/j.1349-7006.2011.01849.x

[40] Akbari, H., Halig, L., Schuster, D.M., Fei, B., Osunkoya, A., Master, V., Nieh, P. and Chen, G. (2012) Hyperspectral Imaging and Quantitative Analysis for Prostate Cancer Detection. Journal of Biomedical Optics, 17, Article ID: 076005. https://doi.org/10.1117/1.JBO.17.7.076005

[41] Melgani, F. and Bruzzone, L. (2004) Classification of Hyperspectral Remote Sensing Images with Support Vector Machines. IEEE Transactions on Geoscience and Remote Sensing, 42, 1778-1790. https://doi.org/10.1109/TGRS.2004.831865

[42] Liu, Z., Yan, J.-Q., Zhang, D. and Li, Q.-L. (2007) Automated Tongue Segmentation in Hyperspectral Images for Medicine. Applied Optics, 46, 8328-8334.

https://doi.org/10.1364/AO.46.008328

[43] Satat, G., Tancik, M. and Raskar, R. (2017) Lensless Imaging with Compressive Ultrafast Sensing. IEEE Transactions on Computational Imaging, 3, 398-407. https://doi.org/10.1109/TCI.2017.2684624

[44] Ekpenyong, N. (2019) Calibration and Characterization of Hyperspectral Imaging Systems Used for Natural Scene Imagery. Optics and Photonics Journal, 9, 81-98. https://doi.org/10.4236/opj.2019.97009

[45] Chandra, M., Scheiman, J., Heidt, D., Simeone, D., McKenna, B. and Mycek, M.-A. (2007) Probing Pancreatic Disease Using Tissue Optical Spectroscopy. Journal of Biomedical Optics, 12, Article ID: 060501. https://doi.org/10.1117/1.2818029

[46] Bradley, R.S. and Thorniley, M.S. (2005) A Review of Attenuation Correction Techniques for Tissue Fluorescence. Journal of the Royal Society Interface, 3, 1-13. https://doi.org/10.1098/rsif.2005.0066 